\title{
Protective Effect of Edaravone against Carbon Monoxide Induced Apoptosis in Rat Primary Cultured Astrocytes
}

\author{
Xiaodan Xu, ${ }^{1}$ Hong Zhang, ${ }^{1}$ Ke Wang, ${ }^{1}$ Tao $\mathrm{Tu},{ }^{2}$ and Yuan Jiang ${ }^{1}$ \\ ${ }^{1}$ Department of Rehabilitation Medicine, The First Affiliated Hospital of Chengdu Medical College, Chengdu 610500, China \\ ${ }^{2}$ Department of Anesthesiology, The First Affiliated Hospital of Chengdu Medical College, Chengdu 610500, China \\ Correspondence should be addressed to Yuan Jiang; 85741920@qq.com
}

Received 22 October 2016; Revised 27 December 2016; Accepted 11 January 2017; Published 2 February 2017

Academic Editor: Angel Catalá

Copyright (c) 2017 Xiaodan Xu et al. This is an open access article distributed under the Creative Commons Attribution License, which permits unrestricted use, distribution, and reproduction in any medium, provided the original work is properly cited.

\begin{abstract}
Objective. To observe the protective effect of edaravone (Eda) on astrocytes after prolonged exposure to carbon monoxide (CO) and further to investigate the potential mechanisms of Eda against CO-induced apoptosis. Methods. The rat primary cultured astrocytes were cultured in vitro and exposed to $1 \% \mathrm{CO}$ for $24 \mathrm{~h}$ after being cultured with different concentrations of Eda. MTT assay was used to detect the cytotoxicity of $\mathrm{CO}$. Flow cytometry was used to detect the apoptosis rate, membrane potential of mitochondria, and ROS level. The mRNA and protein expressions of Bcl-2, Bax, and caspase-3 were assessed by real-time PCR and Western blotting analysis, respectively. Results. Eda can significantly suppress cytotoxicity of CO, and it can significantly increase membrane potential of mitochondria and Bcl-2 expressions and significantly suppress the apoptosis rate, ROS level, Bax, and caspase-3 expressions. Conclusion. Eda protects against CO-induced apoptosis in rat primary cultured astrocytes through decreasing ROS production and subsequently inhibiting mitochondrial apoptosis pathway.
\end{abstract}

\section{Introduction}

Carbon monoxide (CO) is a tasteless, colorless, odorless, and nonirritating but highly toxic gas [1], and it is a by-product of the incomplete combustion of organic material, charcoal burning, domestic heating appliances and systems, and car exhaust. Acute CO poisoning is the most common type of poisoning worldwide, and it accounts for many deaths by accidental, suicide, or otherwise [2]. Inhaled CO mainly is bind with hemoglobin containing $\mathrm{Fe}^{2+}$ to form carboxyhemoglobin $(\mathrm{COHb})[3]$, and $\mathrm{CO}$ has 200 times the affinity for hemoglobin than oxygen that cause impairs oxygen delivery and peripheral utilization leading to tissue hypoxia, oxidative stress, and inflammation within short period (hours or days) after $\mathrm{CO}$ poisoning $[4,5]$. Thus, CO may cause neurological, cerebrovascular, or cardiovascular disorders, such as ischemia, encephalopathy, and peripheral nerve injury.

Most surviving patients can recover completely, but two neurological syndromes may occur in some: permanent neurological sequelae (PNS) and delayed neurological sequelae
(DNS) [6]. About 40\% of surviving patients develop DNS, also known as delayed encephalopathy after acute carbon monoxide poisoning (DEACMP), after a lucid interval from a few days up to months from the acute $\mathrm{CO}$ poisoning. The symptoms of DNS include impaired judgment, poor concentration and memory, cognitive dissonance, personality changes, psychosis, and even Parkinsonism symptoms. Unfortunately, the mechanisms of DNS in humans and in experimental animals remain unclear $[7,8]$. Some previous studies suggested that DNS may be the outcome of acute CO poisoning induced cerebral cellular hypoxia, postischemic reperfusion injury, free radical damage, immune damage, and apoptosis [6]. Free radical damage has important role in postischemic reperfusion injury of cerebral cells after acute $\mathrm{CO}$ poisoning as high concentration of $\mathrm{CO}$ can be combined with the mitochondrial enzyme complex IV which cause accelerated mitochondrial respiratory chain electron leakage leading to excessive ROS production [9]. Astrocytes are the most abundant glial cell types in the brain and play an essential role in the antioxidant defense mechanisms of 
brain tissue. Astrocytes also can provide nutritional and metabolic support to neurons and regulate synaptic activity [10]. High amounts of exogenous CO causes oxidative stress and mitochondrial dysfunction, leading to astrocytic apoptosis and impairment in astrocyte function [11, 12]. Therefore, the inhibition of astrocytic apoptosis may offer protective effects on neurodegenerative disorders after acute $\mathrm{CO}$ poisoning.

Edaravone (Eda) is a novel synthetic small-molecule free radical scavenger [13]. It can protect brain tissue from damage and enhance mental work capacity, due to its high liposolubility and permeability to blood-brain barrier [14]. Thus, it is considered that Eda may be potentially useful for many brain diseases. Some scholars suggested that Eda plays a protective role in the cytotoxicity induced by nitric oxide [15], manganese [16], and neurotoxin MPP+ [13] in astrocytes. However, the protective effects of Eda against CO-induced cytotoxicity in astrocytes are yet to be further investigated. In this present study, we investigated the protective effects of Eda on the CO-induced cytotoxicity in rat primary cultured astrocytes and further explored the potential mechanism involved in the neuroprotective role of Eda.

\section{Materials and Methods}

2.1. Major Reagents. Edaravone injection was purchased from Jiangsu Simcere Pharmaceutical Co., Ltd. (molecular weight: 174.20). Fetal bovine serum (FBS), phosphate buffered solution (PBS), Dulbecco's modified Eagle's medium (DMEM), and trypsin (with phenol red) were purchased from Hyclone (GE Healthcare). MTT cell proliferation and cytotoxicity assay kit, Annexin V-FITC apoptosis detection kit, reactive oxygen species (ROS) assay kit, Rhodamine 123, Trizol, RIPA lysis buffer, enhanced BCA protein assay kit, and SDS-PAGE gel quick preparation kit were purchased from Beyotime. Revert aid first strand cDNA synthesis kit and Super Signal West Pico Chemiluminescent Substrate Trial kit were purchased from Thermo Fisher Scientific Inc. SYBR $^{\circledR}$ Premix Ex Taq ${ }^{\mathrm{TM}}$ II was purchased from Takara Bio Inc. Bcl-2 antibodies, Bax antibodies, caspase- 3 antibodies, and $\beta$-actin antibodies were purchased from Santa Cruz Biotech.

2.2. Cell Culture. The rat primary cultured astrocytes were prepared from cerebral cortex of newborn Sprague-Dawley (SD) rats as previously described [13], and all animal experimental protocol and care were approved by the Institutional Animal Care and Use Committee of Chengdu Medical College. Briefly, after newborn SD rats were killed by rapid decapitation, the cerebral cortices were removed and separated from meninges, blood vessels, and basal ganglia and cut to pieces. The pieces were dissociated with $0.25 \%$ trypsin and terminated by DMEM medium supplemented with $10 \%$ FBS, then centrifugated, and seeded on poly-lysine-coated cell culture flasks (Sigma). The cell culture flasks were placed in incubator at $37^{\circ} \mathrm{C}$ in a humidified atmosphere containing $5 \% \mathrm{CO}_{2}$. After the cell culture flasks have been incubated for $24 \mathrm{~h}$, the culture medium was replaced, and then changed every 2-3 days. The rat primary cultured astrocytes were identified by immunocytochemistry before experiments, and results have shown that more than $98 \%$ of the cells stained positively for the astrocytic marker glial fibrillary acid protein (Sigma).

2.3. Experimental Protocols. The rat primary cultured astrocytes were replated on poly-lysine-coated cell culture 96-well or 6-well plates and randomly divided into control group, CO group, $0.1 \mu \mathrm{M}$ Eda group, $1 \mu \mathrm{M}$ Eda group, $10 \mu \mathrm{M}$ Eda group, and $50 \mu \mathrm{M}$ Eda group. The astrocytes in CO group were exposed to carbon monoxide (the volume fraction is $1 \%$ ) in a sealed chamber containing $5 \% \mathrm{CO}_{2}$ for $24 \mathrm{~h}$ at $37^{\circ} \mathrm{C}$. The $0.1 \mu \mathrm{M}$ Eda group, $1 \mu \mathrm{M}$ Eda group, $10 \mu \mathrm{M}$ Eda group, and $50 \mu \mathrm{M}$ Eda group were preexposed to different concentrations of $\mathrm{Eda}(0.1 \mu \mathrm{M}, 1 \mu \mathrm{M}, 10 \mu \mathrm{M}$, and $50 \mu \mathrm{M})$ for $2 \mathrm{~h}$ and then exposed to $1 \% \mathrm{CO}$ in a sealed chamber containing $5 \%$ $\mathrm{CO}_{2}$ for $24 \mathrm{~h}$ at $37^{\circ} \mathrm{C}$, respectively. The astrocytes in control group were placed in a sealed chamber containing $5 \% \mathrm{CO}_{2}$ for $24 \mathrm{~h}$ at $37^{\circ} \mathrm{C}$.

2.4. MTT Assay. Cytotoxicity of CO was assessed using the 3-(4,5-dimethylthiazol-2-yl)-2,5-diphenyl-tetrazolium bromide (MTT) reduction assay as previously described [17]. Briefly, the astrocytes of different group were seeded into 96-well for $24 \mathrm{~h}$ and then incubated with $200 \mu \mathrm{L}$ MTTcontaining medium $(0.5 \mathrm{mg} / \mathrm{mL}$, diluted with fresh serumfree DMEM medium) for $4 \mathrm{~h}$ at $37^{\circ} \mathrm{C}$ after experimenting. Finally, the MTT-containing medium was removed and $150 \mu \mathrm{L}$ dimethyl sulfoxide (DMSO) was added to each well. The optical density (OD) was measured using an iEMS Analyzer at a wavelength of $570 \mathrm{~nm}$. The cytotoxicity of CO was calculated using the following equation: cytotoxicity (\%) $=\left(\mathrm{OD}_{570}\right.$ control group $-\mathrm{OD}_{570}$ treatment group $) / \mathrm{OD}_{570}$ control group $\times 100$.

2.5. Apoptotic Analysis. Alteration of cell apoptosis level was detected by flow cytometry (FCM) as previously described [17]. Briefly, after being exposed to CO for $24 \mathrm{~h}$, the astrocytes of each group were suspended in the $195 \mu \mathrm{L}$ of $1 \mathrm{x}$ Annexin V-FITC binding buffer and incubated with $5 \mu \mathrm{L}$ of Annexin $\mathrm{V}$-FITC at room temperature (RT) for $10 \mathrm{~min}$; then the astrocytes were centrifuged at $1000 \mathrm{rpm}$ for $5 \mathrm{~min}$. Finally, the astrocytes were resuspended in $190 \mu \mathrm{L}$ of binding buffer and incubated with $10 \mu \mathrm{L}$ of propidium iodide (PI) solution at RT for $5 \mathrm{~min}$. Cell apoptosis level was analyzed using FCM according to the manufacture's instruction.

2.6. Mitochondrial Membrane Functional Measurement. Mitochondrial membrane potential was measured using FCM with Rhodamine 123 staining as previously described [18]. Briefly, after being exposed to CO for $24 \mathrm{~h}$, the astrocytes of each group were incubated with Rhodamine $123(1 \mu \mathrm{M})$ for $30 \mathrm{~min}$ at $37^{\circ} \mathrm{C}$ in the dark. The astrocytes were washed and resuspended in PBS and then analyzed using FCM.

2.7. ROS Measurement. Cellular ROS level was measured using FCM with DCFH-DA kit as previously described [18]. 
Briefly, after being exposed to $\mathrm{CO}$ for $24 \mathrm{~h}$, the astrocytes of each group were incubated with DCFH-DA kit $(10 \mu \mathrm{M})$ for $20 \mathrm{~min}$ at $37^{\circ} \mathrm{C}$ in the dark. The astrocytes were washed and resuspended in PBS and then analyzed using FCM.

2.8. Real-Time PCR Analysis. The mRNA expressions of Bcl2 , Bax, and caspase- 3 were analyzed by real-time PCR as previously described [19]. Briefly, after being exposed to $\mathrm{CO}$ for $24 \mathrm{~h}$, the total RNA of astrocytes of each group were extracted using Trizol reagent according to the manufacturer's instructions and quantified by spectrophotometry at a wavelength of $260 \mathrm{~nm}$. Reverse transcription actions and PCR were performed using reverse transcriptase, oligo (DT) primers, and Taq DNA polymerase. The primers used were as follows: Bcl-2 forward, $5^{\prime}$-TCCGCATCAGGAAGGCTAGA- ${ }^{\prime}$ and reverse, $5^{\prime}$-AGGACCAGGCCTCCAAGCT-3'; Bax forward, $5^{\prime}$-CCTTTTCTACTTTGCCAGCAAAC- $3^{\prime}$ and reverse, 5'-GAGGCCGTCCCAACCAC$3^{\prime}$; caspase- 3 forward, $5^{\prime}$ - AGTCTGACTGGAAAGCCGAA$3^{\prime}$ and reverse, $5^{\prime}$-CGGGATCTGTTTCTTTGCAT- $3^{\prime}$; and GAPDH forward, $5^{\prime}$-CAGGAGGCATTGCTGATGAT- $3^{\prime}$ and reverse, $5^{\prime}$-GAAGGCTGGGGCTCATTT- $3^{\prime}$. The reaction was initiated with denaturation at $95^{\circ} \mathrm{C}$ for $30 \mathrm{sec}$, followed by 40 cycles of $95^{\circ} \mathrm{C}$ for $5 \mathrm{sec}, 60^{\circ} \mathrm{C}$ for $60 \mathrm{sec}$ (annealing), a terminal extension step at $95^{\circ} \mathrm{C}$ for $10 \mathrm{sec}$, and a final holding stage at $4^{\circ} \mathrm{C}$. GAPDH was used as an internal control, and relative mRNA expressions were defined as the ratio of target genes expression to GAPDH expression.

2.9. Western Blot Analysis. The protein expressions of Bcl2 , Bax, and cleaved caspase- 3 were analyzed by Western blot analysis as previously described [19]. Briefly, after being exposed to $\mathrm{CO}$ for $24 \mathrm{~h}$, the total proteins of astrocytes of each group were extracted using RIPA lysis buffer. Protein samples were separated by sodium dodecyl sulfatepolyacrylamide gel electrophoresis and electrotransferred onto a polyvinylidene difluoride (PVDF) membrane. Then, the PVDF membrane was blocked for $2 \mathrm{~h}$ at RT in TBS-Tween 20 (TBST) buffer containing skimmed milk, washed with TBST three times, and incubated overnight at $4^{\circ} \mathrm{C}$ with $1 / 500$ dilution of Bcl-2 antibodies, Bax antibodies, caspase- 3 antibodies, and GAPDH antibodies, respectively. Subsequent to being washed with TBST three times, the PVDF membranes were incubated with horseradish peroxidase-labeled goat anti-rabbit immunoglobulin $\mathrm{G}(\mathrm{H}+\mathrm{L})$ secondary antibody (1/4000 dilution) at $37^{\circ} \mathrm{C}$ for $1 \mathrm{~h}$. Protein signals were detected using SuperSignal West Pico Chemiluminescent Substrate Trial kit and quantified by densitometry using Quantity One software (Bio-Rad).

2.10. Statistical Analysis. The statistical analysis was conducted using SPSS 20.0 for Windows software. Data were present as mean \pm standard deviation (SD). Analysis of variance (ANOVA) was used to compare the differences between groups. Dunnett's T3 was used when the variances were unequal. $P<0.05$ was considered to be statistical significance.

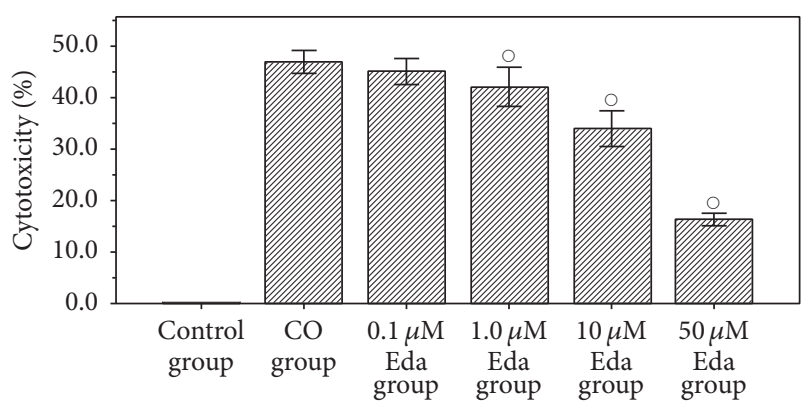

FIGURE 1: The results of cytotoxicity test. Cytotoxicity of each group was assessed using the MTT assay. Compared with $\mathrm{CO}$ group: ${ }^{\circ} \mathrm{P}<$ 0.01 . Five biological replicates were prepared for each sample in this method.

\section{Results}

3.1. MTT Assay. After being preexposed to different concentrations of Eda $(0 \mu \mathrm{M}, 0.1 \mu \mathrm{M}, 1 \mu \mathrm{M}, 10 \mu \mathrm{M}$, and $50 \mu \mathrm{M})$, the astrocytes were treated with $\mathrm{CO}$ for $24 \mathrm{~h}$, and the cytotoxicity of CO was assessed using the MTT assay. As shown in Figure 1, the cytotoxicity of $\mathrm{CO}$ in $\mathrm{CO}$ group was $46.88 \pm$ $1.79 \%$, indicating that $\mathrm{CO}$ has significant direct cytotoxicity to astrocytes. However, the cytotoxicity of $\mathrm{CO}$ in $0.1 \mu \mathrm{M}$ Eda group, $1 \mu \mathrm{M}$ Eda group, $10 \mu \mathrm{M}$ Eda group, and $50 \mu \mathrm{M}$ Eda group was $45.02 \pm 2.03 \%, 43.04 \pm 3.04 \%, 33.92 \pm 2.79 \%$, and $16.31 \pm 0.94 \%$, respectively. By contrast, the cytotoxicity of $\mathrm{CO}$ in $1 \mu \mathrm{M}$ Eda group, $10 \mu \mathrm{M}$ Eda group, and $50 \mu \mathrm{M}$ Eda group was all significantly lower than that in $\mathrm{CO}$ group (all $P<0.01$ ), and the cytotoxicity of CO decreases with drug concentration of Eda.

3.2. Apoptotic Analysis. After being preexposed to different concentrations of Eda $(0 \mu \mathrm{M}, 0.1 \mu \mathrm{M}, 1 \mu \mathrm{M}, 10 \mu \mathrm{M}$, and $50 \mu \mathrm{M})$, the astrocytes were treated with $\mathrm{CO}$ for $24 \mathrm{~h}$, and the alteration of cell apoptosis level was detected by FCM. The results of FCM have shown that (Figure 2), after being exposed to $\mathrm{CO}$ for $24 \mathrm{~h}$, numerous apoptotic cells appeared in the astrocytes of $\mathrm{CO}$ group and the cell apoptosis level was $42.45 \pm 2.36 \%$. It indicted that prolonged exposure to $\mathrm{CO}$ could induce cell apoptosis in astrocytes by direct and strong cytotoxicity. By contrast, the cell apoptosis level of $0.1 \mu \mathrm{M}$ Eda group, $1 \mu \mathrm{M}$ Eda group, $10 \mu \mathrm{M}$ Eda group, and $50 \mu \mathrm{M}$ Eda group was $40.97 \pm 1.32 \%, 36.85 \pm 1.27 \%, 32.64 \pm 2.68 \%$, and $24.9 \pm 3.36 \%$, respectively. The results have shown that, after astrocytes are preexposed to different concentrations of Eda $(0.1 \mu \mathrm{M}, 1 \mu \mathrm{M}, 10 \mu \mathrm{M}$, and $50 \mu \mathrm{M})$, the cytotoxicity activity of CO was decreased, and the cell apoptosis level was gradually declined with drug concentration of Eda, particularly in $1 \mu \mathrm{M}$ Eda group, $10 \mu \mathrm{M}$ Eda group, and $50 \mu \mathrm{M}$ Eda group. The cell apoptosis levels of those groups were all significantly lower than that in CO group (all $P<0.01$ ).

\subsection{Mitochondrial Membrane Functional Measurement.} After being preexposed to different concentrations of Eda $(0 \mu \mathrm{M}, 0.1 \mu \mathrm{M}, 1 \mu \mathrm{M}, 10 \mu \mathrm{M}$, and $50 \mu \mathrm{M})$,the astrocytes were treated with CO for $24 \mathrm{~h}$, and the mitochondrial membrane 


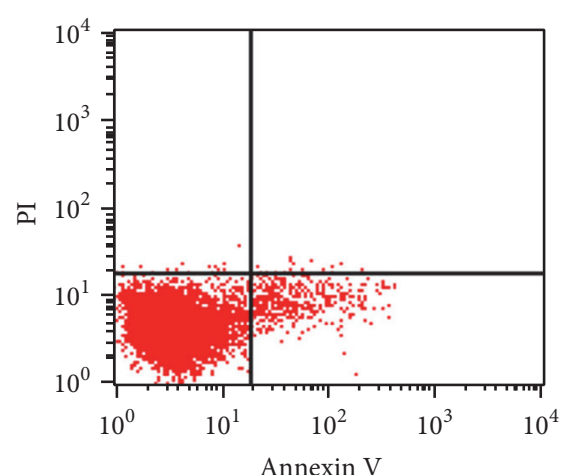

(a)

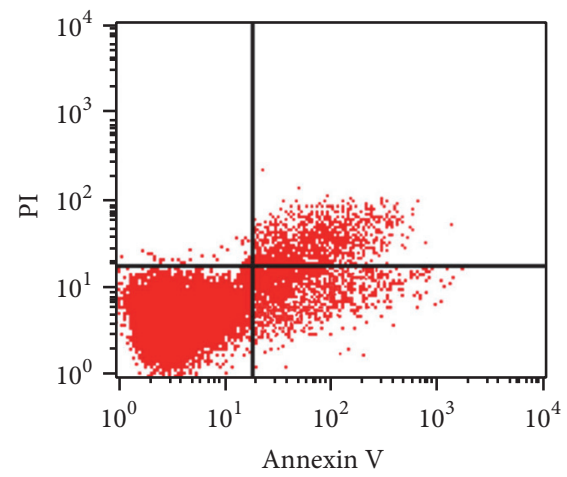

(d)

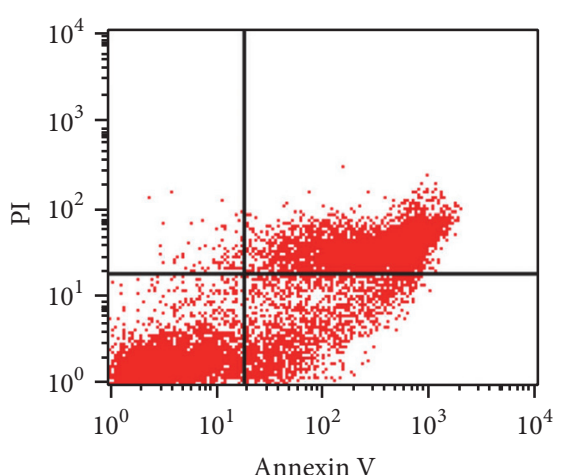

(b)

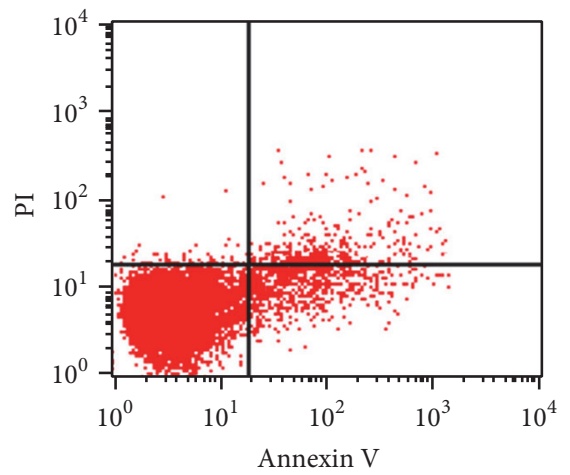

(e)

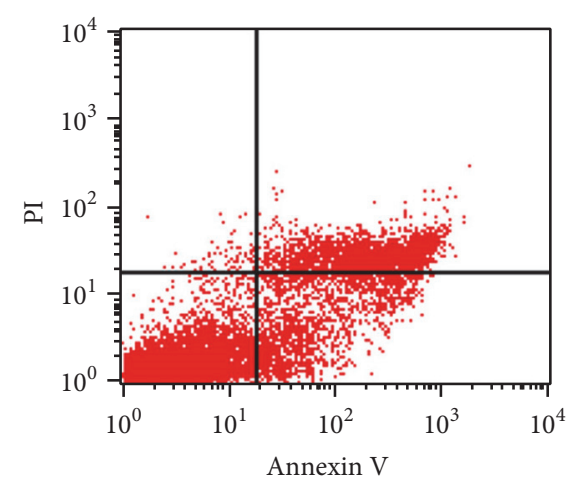

(c)

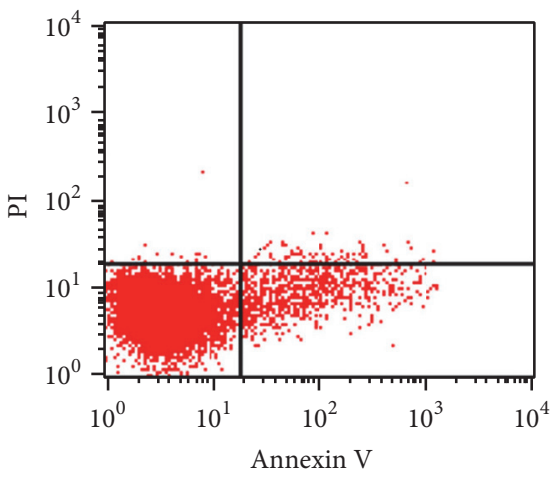

(f)

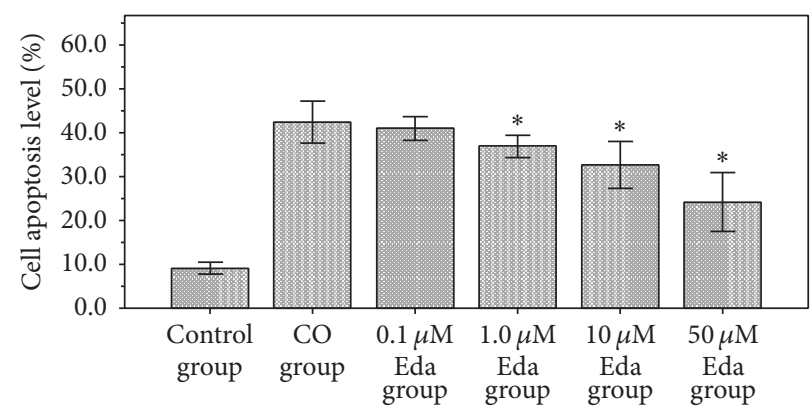

FIgURE 2: The results of apoptotic analysis. Cell apoptosis level of each group was detected by FCM. (a) Control group, (b) CO group, (c) $0.1 \mu \mathrm{M}$ Eda group, (d) $1.0 \mu \mathrm{M}$ Eda group, (e) $10 \mu \mathrm{M}$ Eda group, and (f) $50 \mu \mathrm{M}$ Eda group. Compared with CO group: ${ }^{*} P<0.01$. Three biological replicates were prepared for each sample in this method.

potential was measured using FCM with Rhodamine 123 staining. The results have shown that (Figure 3) the mitochondrial membrane potential of $\mathrm{CO}$ group was significantly lower than control group, indicating that mitochondrial membrane depolarization occurred in the astrocytes treated with $\mathrm{CO}$. Compared with the $\mathrm{CO}$ group, the mitochondrial membrane potential of $1 \mu \mathrm{M}$ Eda group, $10 \mu \mathrm{M}$ Eda group, and $50 \mu \mathrm{M}$ Eda group was significantly increased (all $P<0.01$ ), and the mitochondrial membrane potential increases with drug concentration of Eda.

3.4. ROS Measurement. After being preexposed to different concentrations of $\mathrm{Eda}(0 \mu \mathrm{M}, 0.1 \mu \mathrm{M}, 1 \mu \mathrm{M}, 10 \mu \mathrm{M}$, and $50 \mu \mathrm{M}$ ), the astrocytes were treated with CO for $24 \mathrm{~h}$, and the cellular ROS level was measured using FCM with DCFH-DA kit. As shown in Figure 4(a), in contrast with control group, the fluorescence peak of $\mathrm{CO}$ group was shifted to the right (Figure 4(a)(B)), indicating that the ROS level in the astrocytes after being exposed to $\mathrm{CO}$ for $24 \mathrm{~h}$ was markedly higher than the controlled astrocytes, and prolonged exposure to $\mathrm{CO}$ induced a large generation of ROS. However, after being preexposed to different concentrations of Eda, the ROS levels in the astrocytes treated with $\mathrm{CO}$ for $24 \mathrm{~h}$ were decreased with different degrees.

3.5. Real-Time PCR Analysis. The relative mRNA expressions of target genes were estimated using real-time PCR analysis. As shown in Figure 4(b), in contrast with control group, the mRNA expression of Bcl-2 was significantly decreased $(P<0.01)$ and the mRNA expressions of Bax and caspase3 were significantly increased (all $P<0.01$ ) in CO group. By contrast, after being preexposed to different concentrations of 


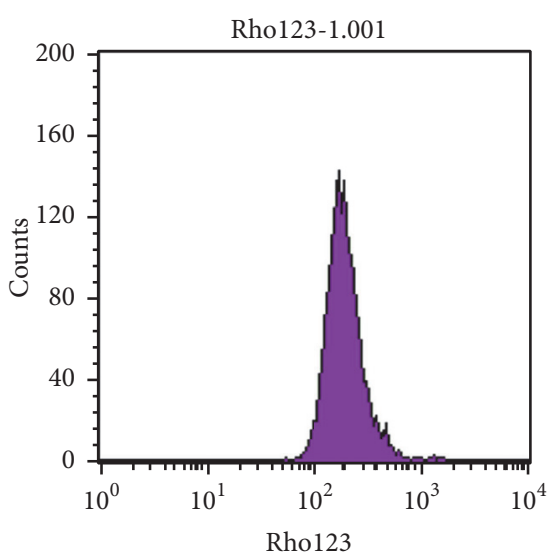

(a)

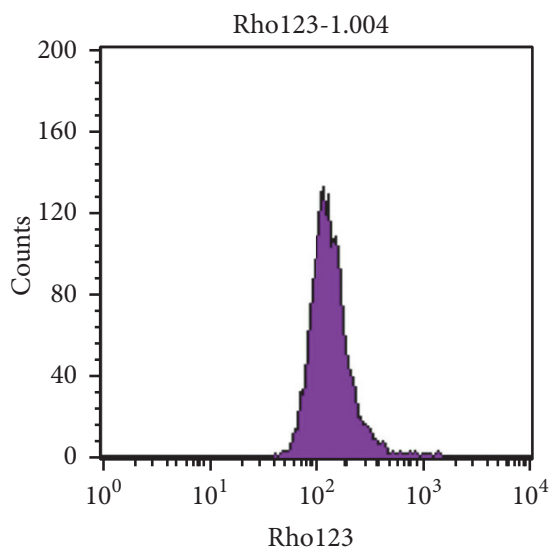

(d)

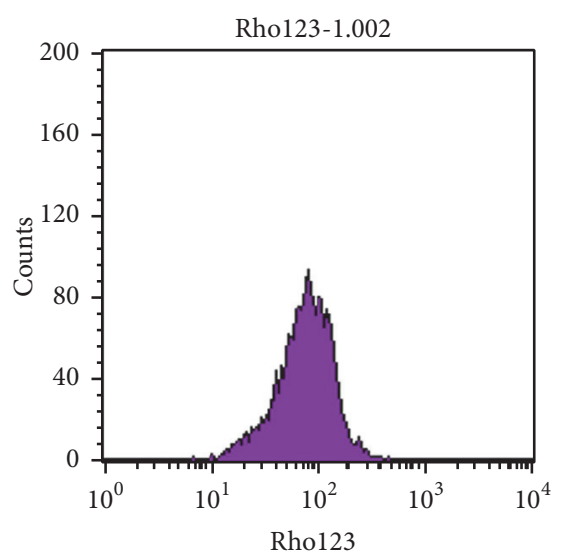

(b)

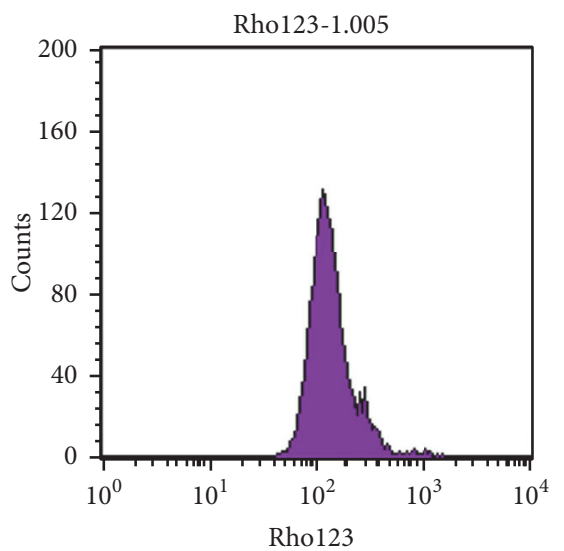

(e)

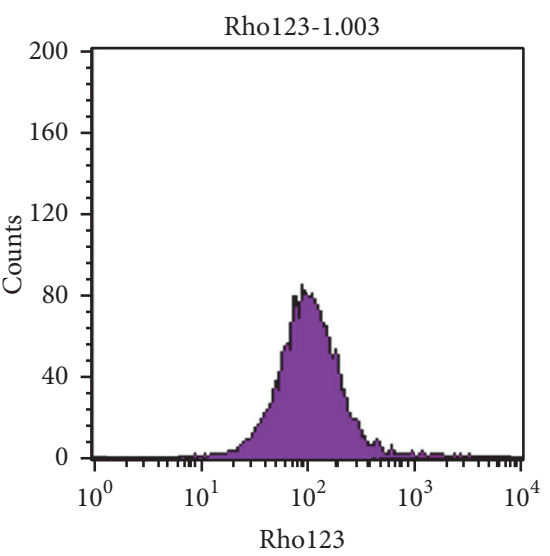

(c)

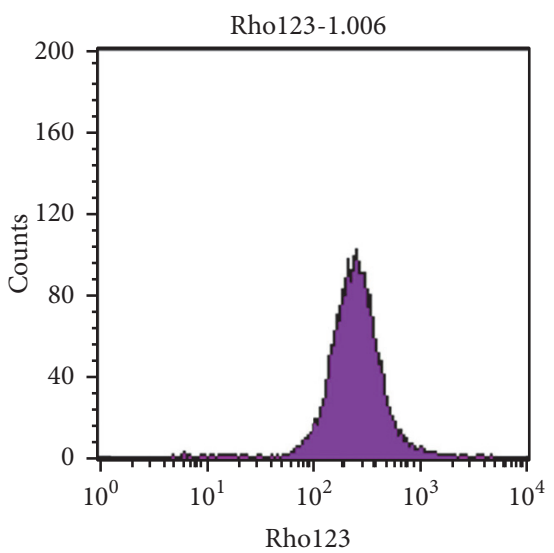

(f)

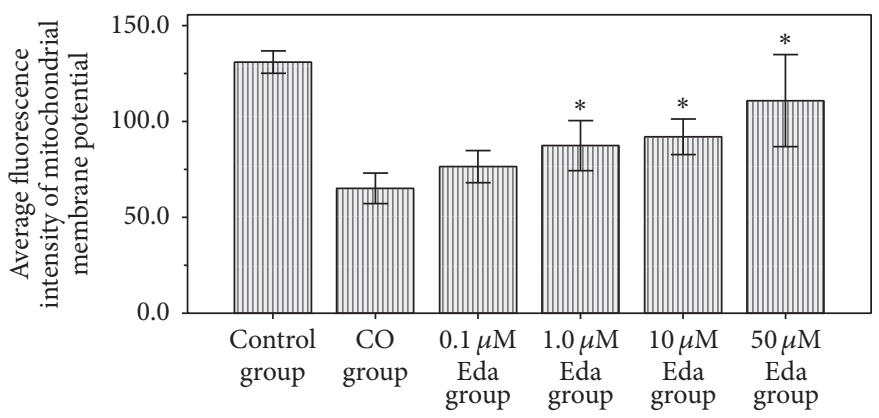

FIGURE 3: The results of mitochondrial membrane functional measurement. Mitochondrial membrane potential of each group was measured using FCM with Rhodamine 123 staining. (a) Control group, (b) CO group, (c) $0.1 \mu \mathrm{M}$ Eda group, (d) $1.0 \mu \mathrm{M}$ Eda group, (e) $10 \mu \mathrm{M}$ Eda group, and (f) $50 \mu \mathrm{M}$ Eda group. Compared with CO group: ${ }^{*} P<0.01$. Three biological replicates were prepared for each sample in this method.

Eda, the mRNA expressions of Bcl-2 were increased and the mRNA expressions of Bax and caspase-3 were decreased with different degrees in the astrocytes treated with $\mathrm{CO}$ for $24 \mathrm{~h}$.

3.6. Western Blot Analysis. The protein expressions of target genes were estimated using Western blot analysis. As shown in Figure 4(c), the protein expression of Bcl-2 was significantly decreased $(P<0.01)$ and the protein expressions of Bax and cleaved caspase- 3 were significantly increased (all $P<0.01$ ) in CO group compared with control group. In contrast with $\mathrm{CO}$ group, after being preexposed to different concentrations of Eda, the protein expressions of Bcl-2 were increased and the protein expressions of Bax and cleaved caspase- 3 were decreased with different degrees in the astrocytes treated with CO for $24 \mathrm{~h}$.

\section{Discussion}

$\mathrm{CO}$ poisoning is one of the common clinical emergencies. Once our bodies had inhaled CO, hemoglobin containing $\mathrm{Fe}^{2+}$ were bound with $\mathrm{CO}$ to form $\mathrm{COHb}$, leading to oxygen deprivation, in particular, brain tissue hypoxia. Irreversible 


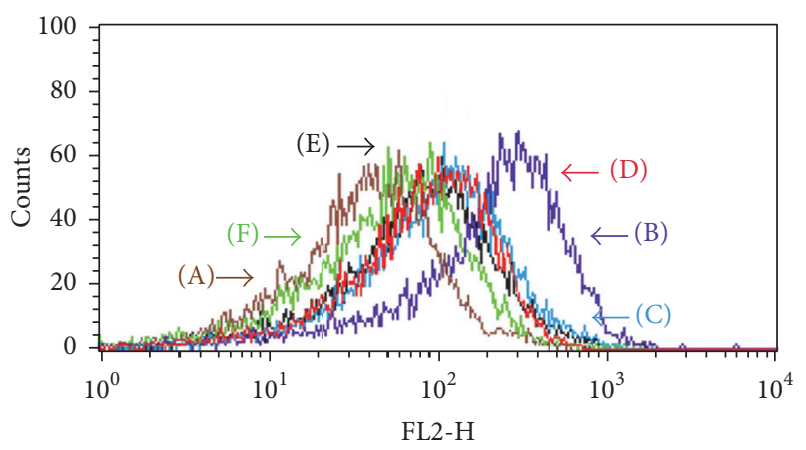

(a)

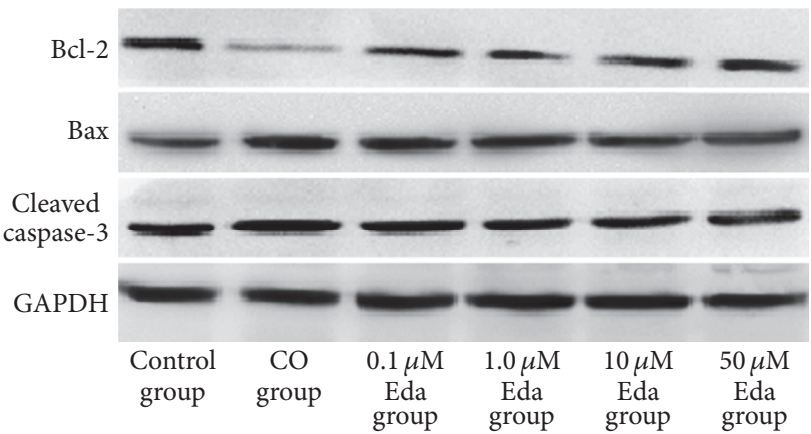

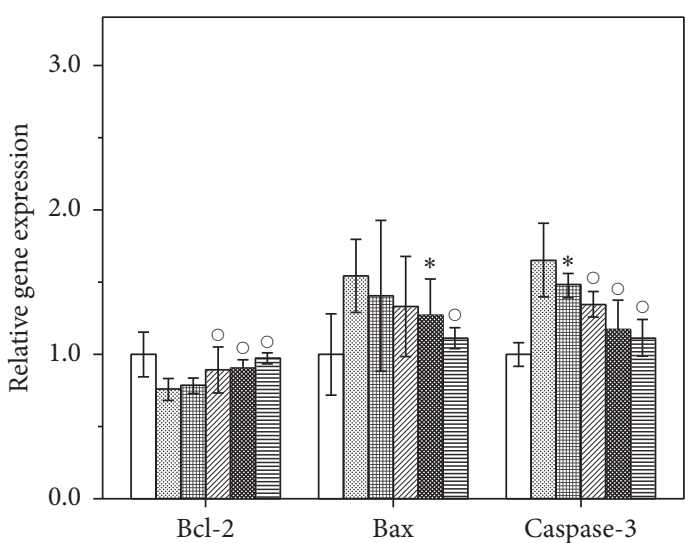

Groups

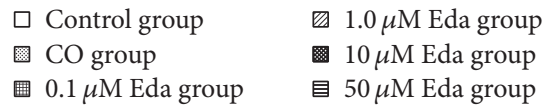

(b)

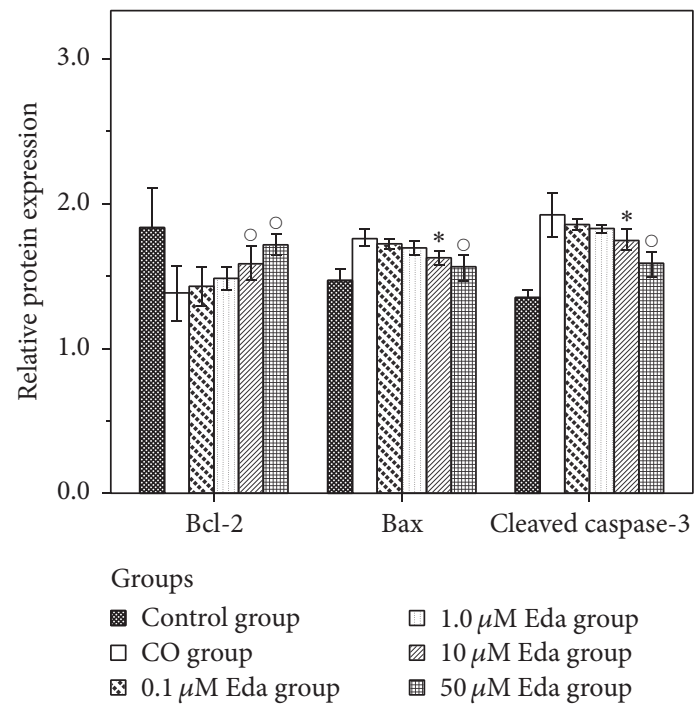

(c)

FIgURE 4: The results of ROS measurement, real-time PCR analysis, and Western blot analysis. (a) Cellular ROS level of each group was measured using FCM with DCFH-DA kit. (A) Control group, (B) CO group, (C) $0.1 \mu \mathrm{M}$ Eda group, (D) $1.0 \mu \mathrm{M}$ Eda group, (E) $10 \mu \mathrm{M}$ Eda group, and (F) $50 \mu \mathrm{M}$ Eda group. (b) The relative mRNA expressions of Bcl-2, Bax, and cleaved caspase-3 were estimated using real-time PCR analysis. Compared with CO group: ${ }^{*} \mathrm{P}<0.05,{ }^{\circ} \mathrm{P}<0.01$. (c) The protein expressions of Bcl-2, Bax, and caspase- 3 were estimated using Western blot analysis. Compared with CO group: ${ }^{*} P<0.05,{ }^{\circ} \mathrm{P}<0.01$. For each method, three biological replicates were prepared for each sample.

injury of brain neurons results from the context of prolonged exposure to CO [20]. DNS is a kind of delayed encephalopathy symptoms that occur in surviving patients after acute $\mathrm{CO}$ poisoning for a period of days to weeks [6]. In the period of time from before DNS occur in surviving patients, the clinical symptoms seem to be cured by effective symptomatic treatment, but DNS may occur after inadequate continuous treatment. Therefore, to investigate the mechanisms of DNS is greatly significant for effective prevention and treatment of DNS in clinic. Recent studies showed that cerebral cellular hypoxia, postischemic reperfusion injury, free radical damage, immune damage, and apoptosis may involve in the mechanisms of DNS [6], and it was considered that free radical damage and apoptosis were presumed to be one of the main pathogenesis. Astrocytes are important for the survival of neurons and defense effect of the central nervous system and play an important role in the process of scavenging oxygen free radicals in brain tissue [21]. Astrocytic apoptosis and dysfunction of astrocytes can negatively affect neuronal survival and may contribute to the pathogenesis of many brain diseases. In the present study, the results of MTT assay showed that the cytotoxicity of CO induces the death of astrocytes. Of course, $\mathrm{CO}$ can suppress the activity of succinate dehydrogenase which combined with mitochondrial inner 
membrane to partly affect the results of OD in MTT assay. However, it indirectly indicates that CO can damage mitochondrial respiratory chain function through inactivation of succinate dehydrogenase and ultimately lead to astrocytes death. Moreover, the results of apoptotic analysis showed that apoptosis was a main death mode of astrocytes after prolonged exposure to $\mathrm{CO}$. The results of ROS and mitochondrial membrane potential measurement showed that vast ROS were generated in the astrocytes and causing mitochondrial membrane potential significantly decreased after a prolonged exposure to $\mathrm{CO}$. $\mathrm{Bcl}-2$ is a tumor suppressing gene, regulating mitochondrial membrane permeability to inhibit the signal transduction pathway of cell apoptosis. Proapoptotic gene Bax can accelerate irreversible apoptosis through disruption of mitochondrial membrane potential and activation of caspase-3. In the present study, we found that the mRNA and protein expressions of Bcl-2 were significantly decreased, and the mRNA and protein expressions of Bax and caspase- 3 were significantly increased in the astrocytes after a prolonged exposure to CO. So we suspected that $\mathrm{CO}$ can induce the damage of mitochondrial membrane by causing the collapse of mitochondrial membrane potential and the generation of ROS that is causing mitochondrial apoptosis pathway activation in cerebral cells and ultimately lead to DNS in acute carbon monoxide poisoning patients. However, of course, $\mathrm{CO}$ has dual effect on modulation of apoptosis in astrocytes depending on its concentration. Almeida et al. [22] suggested that low concentrations of CO partially inhibited oxidative stress-induced apoptosis in astrocytes and played a positive role of protection by preventing mitochondrial potential depolarization and plasmatic membrane permeability, increasing the expressions of $\mathrm{Bcl}-2$ and suppressing caspase- 3 activation. Moreover, CO-induced ROS can become toxic or signaling depending on its concentration as well. Therefore, future investigation of the potential mechanism of different concentrations of $\mathrm{CO}$ in modulating mitochondrial function is necessary to be conducted.

Despite the acute carbon monoxide poisoning patients can receive various forms of treatments in present, hyperbaric oxygen therapy (HBOT) is the first choice and performing HBOT as early as possible is preferred, and postoperative prognosis is good. However, the preventive effects of HBOT on DNS remain controversial $[23,24]$. Eda is a free radical scavenger, and it can effectively improve the tolerance of cerebral cells to hypoxia and protect the function of brain in the patients with acute CO poisoning. Thus, Eda can act as a potential therapy for preventing DNS. Li et al. [25] suggested that Eda attenuates brain damage in rats after acute $\mathrm{CO}$ poisoning by inhibiting oxidative stress and neuronal apoptosis. However, the protective mechanism of Eda against $\mathrm{CO}$-induced cytotoxicity in astrocytes remains unclear. In the present study, after being preexposed to different concentrations of Eda $(0.1 \mu \mathrm{M}, 1 \mu \mathrm{M}, 10 \mu \mathrm{M}$, and $50 \mu \mathrm{M})$, the cytotoxicity of $\mathrm{CO}$ and the apoptosis level gradually decline with drug concentration of Eda in astrocytes, indicating that Eda can effectively resist the cell damage caused by prolonged exposure to $\mathrm{CO}$ and has a protective effect on astrocytes. The results of ROS and mitochondrial membrane potential measurement showed that Eda can effectively eliminate ROS and attenuate the damage of mitochondrial membrane in astrocytes. Moreover, the results of mRNA and protein expressions of Bcl-2, Bax, and caspase-3 were indicating that Eda inhibited mitochondrial apoptosis pathway activation by increasing the expressions of $\mathrm{Bcl}-2$ and suppressing the expressions of Bax and caspase-3. Therefore, we suggested that the protective mechanism of Eda may contribute to inhibiting mitochondrial apoptosis pathway activation by effectively scavenging ROS and protecting the function of mitochondria.

In conclusion, this study firstly reveals that Eda protects against $\mathrm{CO}$-induced apoptosis in rat primary cultured astrocytes through decreasing ROS production and subsequently inhibiting mitochondrial apoptosis pathway. Eda appears to be effective for the treatment of patients with acute $\mathrm{CO}$ poisoning, and it can be useful in the prevention and treatment of DNS.

$\begin{array}{ll}\text { Abbreviations } \\ \text { Eda: } & \text { Edaravone } \\ \text { CO: } & \text { Carbon monoxide } \\ \text { MTT: } & \begin{array}{l}\text { 3-(4,5-Dimethylthiazol-2-yl)-2,5- } \\ \text { diphenyl-tetrazolium }\end{array} \\ & \text { bromide } \\ \text { COHb: } & \text { Carboxyhemoglobin } \\ \text { PNS: } & \text { Permanent neurological sequelae } \\ \text { DEACMP: } & \text { Acute carbon monoxide poisoning } \\ \text { DNS: } & \text { Delayed neurological sequelae } \\ \text { ROS: } & \text { Reactive oxygen species } \\ \text { FBS: } & \text { Fetal bovine serum } \\ \text { PBS: } & \text { Phosphate buffered solution } \\ \text { DMEM: } & \text { Dulbeccos modified Eagle's medium } \\ \text { SD: } & \text { Sprague-Dawley } \\ \text { DMSO: } & \text { Dimethyl sulfoxide } \\ \text { OD: } & \text { Optical density } \\ \text { FCM: } & \text { Flow cytometry } \\ \text { RT: } & \text { Room temperature } \\ \text { PI: } & \text { Propidium iodide } \\ \text { PVDF: } & \text { Polyvinylidene difluoride } \\ \text { HBOT: } & \text { Hyperbaric oxygen therapy. } \\ & \end{array}$

\section{Competing Interests}

The authors declare no conflict of interests

\section{Acknowledgments}

This work was supported by the grants from Sichuan Province Health Department of Scientific Research Subject of China (no. 130383). The authors also express their sincere thanks to Dr. Yi Yuan, Miss. Xiaofei Wei, and Ms. Zunzhen Zhou for their helpful assistance.

\section{References}

[1] T. Tanaka, T. Kashimura, M. Ise, B. D. Lohman, and Y. Taira, "Light irradiation for treatment of acute carbon monoxide poisoning: an experimental study," Journal of Intensive Care, vol. 4, no. 1, 2016. 
[2] S. Iqbal, J. H. Clower, S. A. Hernandez, S. A. Damon, and F. Y. Yip, "A review of disaster-related carbon monoxide poisoning: surveillance, epidemiology, and opportunities for prevention," American Journal of Public Health, vol. 102, no. 10, pp. 1957-1963, 2012.

[3] L. R. Goldbaum, R. G. Ramirez, and K. B. Absalon, "What is the mechanism of carbon monoxide toxicity," Aviation Space and Environmental Medicine, vol. 46, no. 10, pp. 1289-1291, 1975.

[4] R. E. Ghosh, R. Close, L. J. McCann et al., "Analysis of hospital admissions due to accidental non-fire-related carbon monoxide poisoning in England, between 2001 and 2010," Journal of Public Health, vol. 38, no. 1, pp. 76-83, 2016.

[5] M. L. Bleecker, "Carbon monoxide intoxication," Handbook of Clinical Neurology, vol. 131, pp. 191-203, 2015.

[6] W. Li, Y. Zhang, R. Gu et al., "DNA pooling base genomewide association study identifies variants at NRXN3 associated with delayed encephalopathy after acute carbon monoxide poisoning," PLoS ONE, vol. 8, no. 11, Article ID e79159, 2013.

[7] Y. C. Chang, H. Y. Lee, J. L. Huang, C. H. Chiu, C. L. Chen, and C. T. Wu, "Risk factors and outcome analysis in children with carbon monoxide poisoning," Pediatrics \& Neonatology, 2016.

[8] D. Taskiran, T. Nesil, and K. Alkan, "Mitochondrial oxidative stress in female and male rat brain after ex vivo carbon monoxide treatment," Human and Experimental Toxicology, vol. 26, no. 8, pp. 645-651, 2007.

[9] G. P. Roberts, H. Youn, and R. L. Kerby, "CO-sensing mechanisms," Microbiology and Molecular Biology Reviews, vol. 68, no. 3, pp. 453-473, 2004.

[10] M. Uno, K. T. Kitazato, A. Suzue et al., "Inhibition of brain damage by edaravone, a free radical scavenger, can be monitored by plasma biomarkers that detect oxidative and astrocyte damage in patients with acute cerebral infarction," Free Radical Biology and Medicine, vol. 39, no. 8, pp. 1109-1116, 2005.

[11] D. M. Jurič, Ž. Finderle, D. Šuput, and M. Brvar, "The effectiveness of oxygen therapy in carbon monoxide poisoning is pressure- and time-dependent: a study on cultured astrocytes," Toxicology Letters, vol. 233, no. 1, pp. 16-23, 2015.

[12] D. M. Jurič, D. Šuput, and M. Brvar, "Hyperbaric oxygen preserves neurotrophic activity of carbon monoxide-exposed astrocytes," Toxicology Letters, vol. 253, pp. 1-6, 2016.

[13] H. Chen, S. Wang, J.-H. Ding, and G. Hu, "Edaravone protects against $\mathrm{MPP}^{+}$-induced cytotoxicity in rat primary cultured astrocytes via inhibition of mitochondrial apoptotic pathway," Journal of Neurochemistry, vol. 106, no. 6, pp. 2345-2352, 2008.

[14] P. Zhang, W. Li, L. Li et al., "Treatment with edaravone attenuates ischemic brain injury and inhibits neurogenesis in the subventricular zone of adult rats after focal cerebral ischemia and reperfusion injury," Neuroscience, vol. 201, pp. 297-306, 2012.

[15] T. Kawasaki, T. Kitao, K. Nakagawa et al., "Nitric oxide-induced apoptosis in cultured rat astrocytes: protection by edaravone, a radical scavenger," GLIA, vol. 55, no. 13, pp. 1325-1333, 2007.

[16] V. Evren, M. Apaydin, A. Khalilnezhad, O. Erbas, and D. Taskiran, "Protective effect of edaravone against manganeseinduced toxicity in cultured rat astrocytes," Environmental Toxicology \& Pharmacology, vol. 40, no. 2, pp. 563-567, 2015.

[17] Y. Jiang, X. Xia, A. W. Leung, J. Xiang, and C. Xu, "Apoptosis of breast cancer cells induced by hypocrellin B under lightemitting diode irradiation," Photodiagnosis and Photodynamic Therapy, vol. 9, no. 4, pp. 337-343, 2012.
[18] J. Ying, H. Xu, D. Wu, and X. Wu, "Emodin induces apoptosis of human osteosarcoma cells via mitochondria- and endoplasmic reticulum stress-related pathways," International Journal of Clinical and Experimental Pathology, vol. 8, no. 10, pp. 1283712844, 2015.

[19] Y. Jiang, H. Gou, J. Zhu, S. Tian, and L. Yu, "Lidocaine inhibits the invasion and migration of TRPV6-expressing cancer cells by TRPV6 downregulation," Oncology Letters, vol. 12, no. 2, pp. 1164-1170, 2016.

[20] L. D. Prockop and R. I. Chichkova, "Carbon monoxide intoxication: an updated review," Journal of the Neurological Sciences, vol. 262, no. 1-2, pp. 122-130, 2007.

[21] C. Giaume, F. Kirchhoff, C. Matute, A. Reichenbach, and A. Verkhratsky, "Glia: the fulcrum of brain diseases," Cell Death and Differentiation, vol. 14, no. 7, pp. 1324-1335, 2007.

[22] A. S. Almeida, C. S. F. Queiroga, M. F. Q. Sousa, P. M. Alves, and H. L. A. Vieira, "Carbon monoxide modulates apoptosis by reinforcing oxidative metabolism in astrocytes: role of Bcl-2," The Journal of Biological Chemistry, vol. 287, no. 14, pp. 1076110770, 2012.

[23] G. Speit, C. Dennog, P. Radermacher, and A. Rothfuss, "Genotoxicity of hyperbaric oxygen," Mutation Research/Reviews in Mutation Research, vol. 512, no. 2-3, pp. 111-119, 2002.

[24] M. Gröger, P. Radermacher, G. Speit, and C.-M. Muth, “Genotoxicity of hyperbaric oxygen and its prevention: what hyperbaric physicians should know," Diving and Hyperbaric Medicine, vol. 38, no. 4, pp. 200-205, 2008.

[25] Q. Li, M. J. Bi, W. K. Bi, H. Kang, L. J. Yan, and Y.-L. Guo, "Edaravone attenuates brain damage in rats after acute $\mathrm{CO}$ poisoning through inhibiting apoptosis and oxidative stress," Environmental Toxicology, vol. 31, no. 3, pp. 372-379, 2016. 

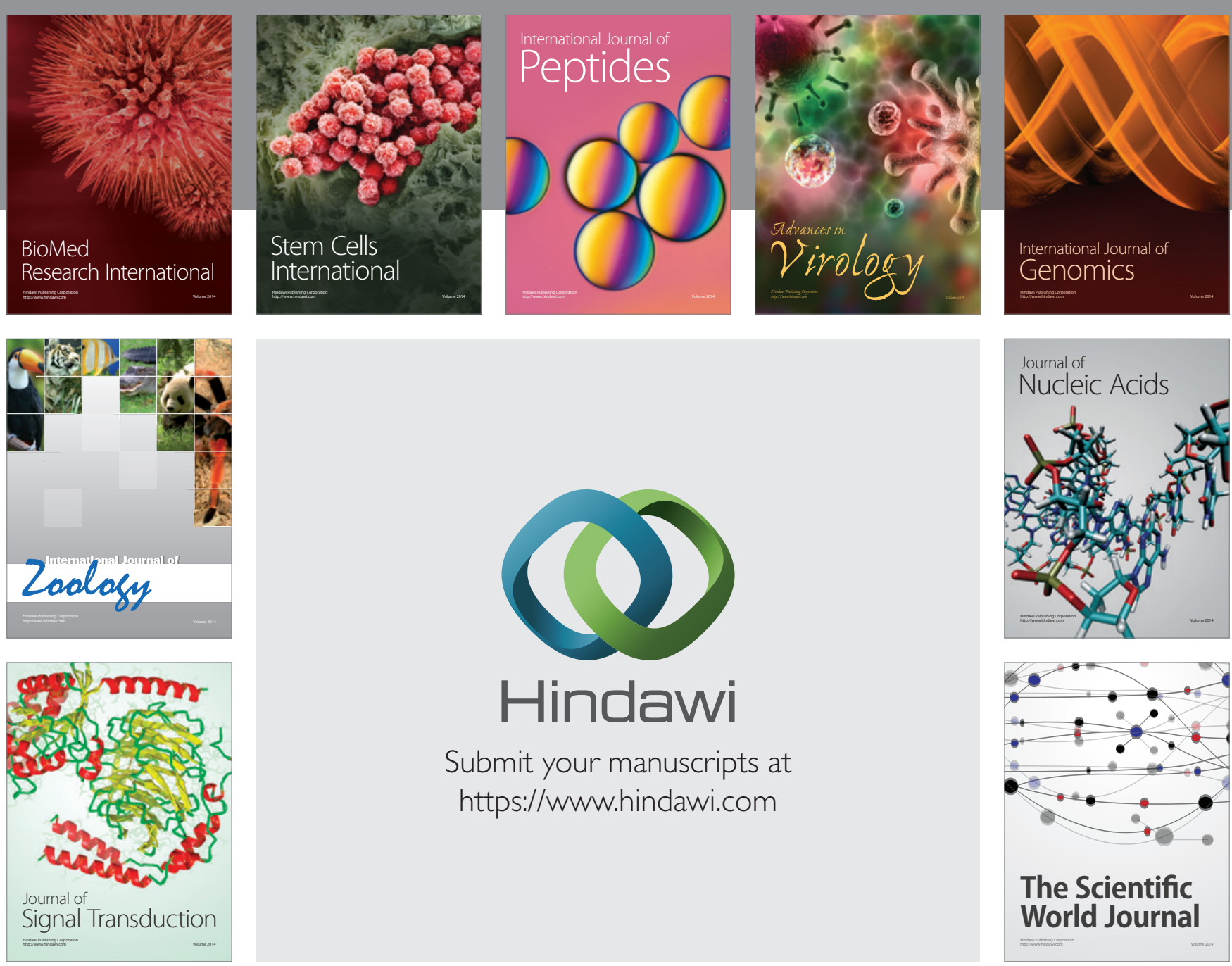

Submit your manuscripts at

https://www.hindawi.com
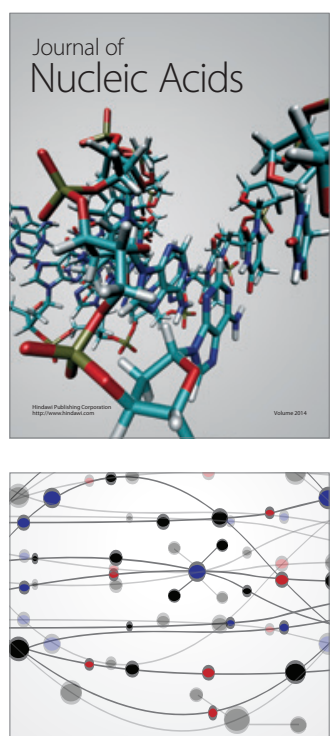

The Scientific World Journal
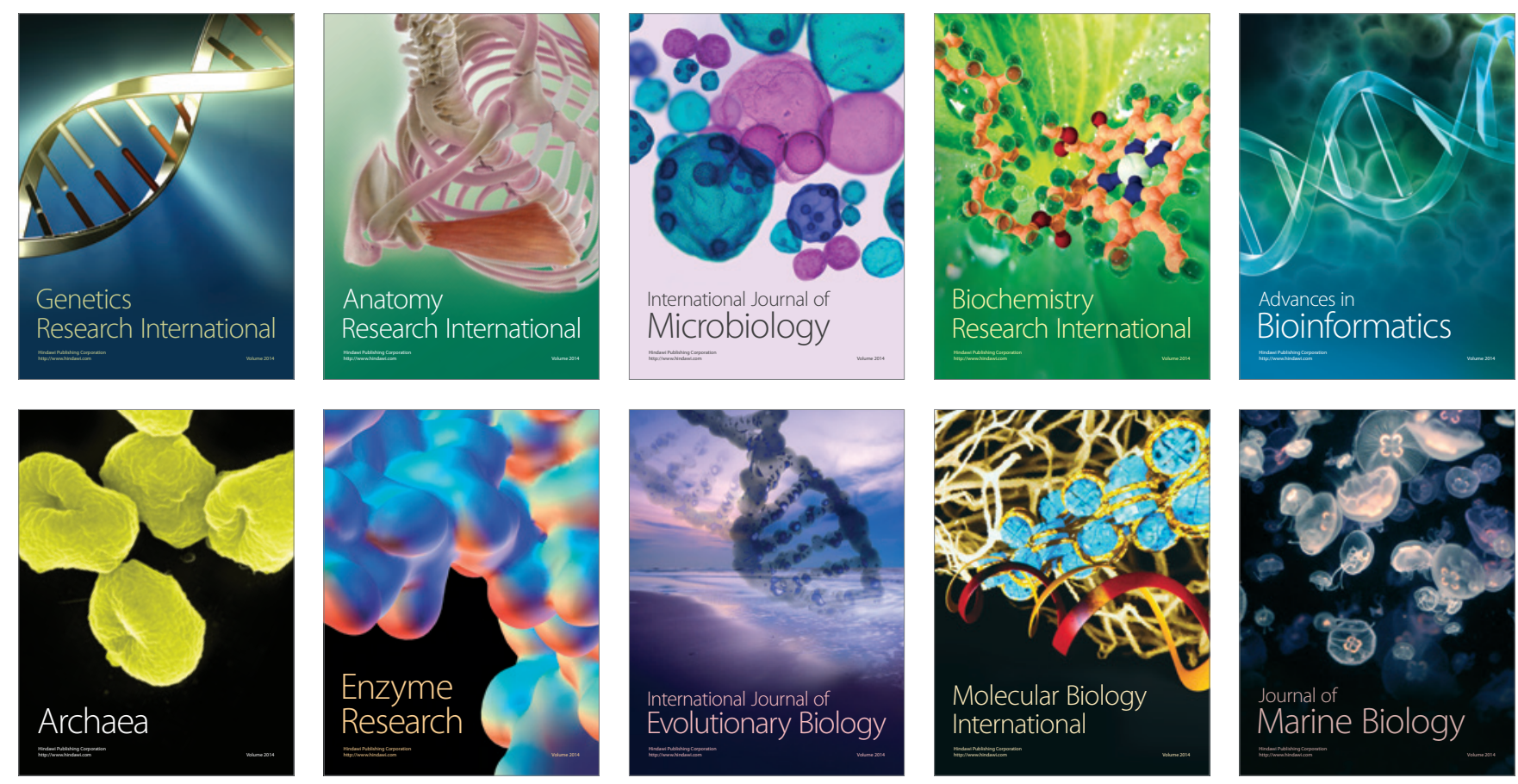